\title{
HYERS-ULAM STABILITY OF BUTLER-RASSIAS FUNCTIONAL EQUATION
}

SOON-MO JUNG

Received 10 June 2004

We will prove the Hyers-Ulam stability of the Butler-Rassias functional equation following an idea by M. T. Rassias.

\section{Introduction}

In 1940, Ulam [9] gave a wide ranging talk before the Mathematics Club of the University of Wisconsin in which he discussed a number of important unsolved problems. Among those was the following question concerning the stability of homomorphisms.

Let $G_{1}$ be a group and let $G_{2}$ be a metric group with a metric $d(\cdot, \cdot)$. Given $\varepsilon>0$, does there exist a $\delta>0$ such that if a function $h: G_{1} \rightarrow G_{2}$ satisfies the inequality $d(h(x y), h(x) h(y))<\delta$ for all $x, y \in G_{1}$, then a homomorphism $H: G_{1} \rightarrow G_{2}$ exists with $d(h(x), H(x))<\varepsilon$ for all $x \in$ $G_{1}$ ?

The case of approximately additive functions was solved by Hyers [5] under the assumption that $G_{1}$ and $G_{2}$ are Banach spaces.

Taking this fact into account, the additive Cauchy functional equation $f(x+y)=$ $f(x)+f(y)$ is said to have the Hyers-Ulam stability. This terminology is also applied to the case of other functional equations. For a more detailed definition of such terminology, one can refer to $[4,6,7]$.

In 2003, Butler [3] posed the following problem.

Problem 1.1 (Butler [3]). Show that for $d<-1$, there are exactly two solutions $f: \mathbb{R} \rightarrow \mathbb{R}$ of the functional equation $f(x+y)-f(x) f(y)=d \sin x \sin y$.

Recently, Rassias excellently answered this problem by proving the following theorem (see [8]).

Theorem 1.2 (Rassias [8]). Let $d<-1$ be a constant. The functional equation

$$
f(x+y)-f(x) f(y)=d \sin x \sin y
$$


has exactly two solutions in the class of functions $f: \mathbb{R} \rightarrow \mathbb{R}$. More precisely, if a function $f: \mathbb{R} \rightarrow \mathbb{R}$ satisfies the Butler-Rassias functional equation for all $x, y \in \mathbb{R}$, then $f$ has one of the forms

$$
f(x)=c \sin x+\cos x, \quad f(x)=-c \sin x+\cos x
$$

where $c=\sqrt{-d-1}$ is set.

In this paper, we will prove the Hyers-Ulam stability of the Butler-Rassias functional equation (1.1).

\section{Preliminaries}

We follow an idea of Rassias [8] to prove the following lemma. In Section 3, we apply this lemma to the proof of the Hyers-Ulam stability of the Butler-Rassias functional equation (1.1).

LEMma 2.1. Let $d$ be a nonzero real number and $0<\varepsilon<|d|$. If a function $f: \mathbb{R} \rightarrow \mathbb{R}$ satisfies the functional inequality

$$
|f(x+y)-f(x) f(y)-d \sin x \sin y| \leq \varepsilon
$$

for all $x, y \in \mathbb{R}$, then $M_{f}:=\sup _{x \in \mathbb{R}}|f(x)|$ is finite and

$$
\left|f(x)-f\left(\frac{\pi}{2}\right) \sin x-\cos x\right| \leq \frac{2\left(1+M_{f}\right)}{|d|} \varepsilon
$$

for all $x \in \mathbb{R}$.

Proof. If we replace $x$ by $x+z$ in (2.1), then we have

$$
|f(x+y+z)-f(x+z) f(y)-d \sin (x+z) \sin y| \leq \varepsilon
$$

for any $x, y, z \in \mathbb{R}$. Similarly, if we replace $y$ by $y+z$ in (2.1), then we get

$$
|f(x+y+z)-f(x) f(y+z)-d \sin x \sin (y+z)| \leq \varepsilon
$$

for $x, y, z \in \mathbb{R}$.

Using (2.3) and (2.4), we obtain

$$
\begin{gathered}
|f(x) f(y+z)-f(x+z) f(y)+d \sin x \sin (y+z)-d \sin (x+z) \sin y| \\
=\mid[f(x+y+z)-f(x+z) f(y)-d \sin (x+z) \sin y] \\
\quad-[f(x+y+z)-f(x) f(y+z)-d \sin x \sin (y+z)] \mid \leq 2 \varepsilon
\end{gathered}
$$


for all $x, y, z \in \mathbb{R}$. It follows from (2.5) that

$$
\begin{aligned}
& \mid f(x)[f(y+z)-f(y) f(z)-d \sin y \sin z]+f(x) f(y) f(z)+d f(x) \sin y \sin z \\
& -[f(x+z)-f(x) f(z)-d \sin x \sin z] f(y)-f(x) f(y) f(z)-d f(y) \sin x \sin z \\
& +d \sin x \sin (y+z)-d \sin (x+z) \sin y \mid \\
& \quad=|f(x) f(y+z)-f(x+z) f(y)+d \sin x \sin (y+z)-d \sin (x+z) \sin y| \leq 2 \varepsilon
\end{aligned}
$$

for all $x, y, z \in \mathbb{R}$.

It is easy to check that

$$
\begin{aligned}
&|d f(x) \sin y \sin z+d \sin x \sin (y+z)-d f(y) \sin x \sin z-d \sin (x+z) \sin y| \\
&=\mid f(x)[f(y+z)-f(y) f(z)-d \sin y \sin z] \\
&+f(x) f(y) f(z)+d f(x) \sin y \sin z \\
&-[f(x+z)-f(x) f(z)-d \sin x \sin z] f(y) \\
&-f(x) f(y) f(z)-d f(y) \sin x \sin z \\
&+d \sin x \sin (y+z)-d \sin (x+z) \sin y \\
&-f(x)[f(y+z)-f(y) f(z)-d \sin y \sin z] \\
&+[f(x+z)-f(x) f(z)-d \sin x \sin z] f(y) \mid .
\end{aligned}
$$

Hence, in view of (2.6) and (2.1), we can now get

$$
\begin{aligned}
& |d f(x) \sin y \sin z+d \sin x \sin (y+z)-d f(y) \sin x \sin z-d \sin (x+z) \sin y| \\
& \leq \mid f(x)[f(y+z)-f(y) f(z)-d \sin y \sin z] \\
& \quad+f(x) f(y) f(z)+d f(x) \sin y \sin z \\
& \quad-[f(x+z)-f(x) f(z)-d \sin x \sin z] f(y) \\
& \quad-f(x) f(y) f(z)-d f(y) \sin x \sin z \\
& \quad+d \sin x \sin (y+z)-d \sin (x+z) \sin y \mid \\
& +|f(x)||f(y+z)-f(y) f(z)-d \sin y \sin z| \\
& +|f(y)||f(x+z)-f(x) f(z)-d \sin x \sin z| \\
& \leq \\
& \quad(2+|f(x)|+|f(y)|) \varepsilon
\end{aligned}
$$

for all $x, y, z \in \mathbb{R}$. If we set $y=z=\pi / 2$ in the above inequality, then

$$
\left|d f(x)-d f\left(\frac{\pi}{2}\right) \sin x-d \cos x\right| \leq\left(2+|f(x)|+\left|f\left(\frac{\pi}{2}\right)\right|\right) \varepsilon
$$

for each $x \in \mathbb{R}$.

If we assume that $f$ were unbounded, there should exist a sequence $\left\{x_{n}\right\} \subset \mathbb{R}$ such that $f\left(x_{n}\right) \neq 0$ for every $n \in \mathbb{N}$ and $\left|f\left(x_{n}\right)\right| \rightarrow \infty$ as $n \rightarrow \infty$. Set $x=x_{n}$ in (2.9), divide both sides of the resulting inequality by $\left|f\left(x_{n}\right)\right|$, and then let $n$ diverge to infinity. Then, we have $|d| \leq \varepsilon$ which is contrary to our hypothesis, say $\varepsilon<|d|$. 
Therefore, $f$ must be bounded, and hence $M_{f}:=\sup _{x \in \mathbb{R}}|f(x)|$ has to be finite. Therefore, it follows from (2.9) that (2.2) holds for each $x \in \mathbb{R}$.

\section{Hyers-Ulam stability}

In this section, using Lemma 2.1, we prove the Hyers-Ulam stability of the Butler-Rassias functional equation.

Theorem 3.1. Let $d<-1$ be a constant. Then there exists a constant $K=K(d) \geq 0$ such that if $0<\varepsilon<|d|$ and if a function $f: \mathbb{R} \rightarrow \mathbb{R}$ satisfies the functional inequality (2.1) for all $x, y \in \mathbb{R}$, then

$$
\left|f(x)-f_{0}(x)\right| \leq K(\varepsilon+\sqrt{\varepsilon})
$$

holds for all $x \in \mathbb{R}$ and for some solution function $f_{0}$ of the Butler-Rassias functional equation.

Proof. Let $0<\varepsilon<|d|$ and $f$ a real-valued function on $\mathbb{R}$ which satisfies inequality (2.1). It follows from Lemma 2.1 that $M_{f}:=\sup _{x \in \mathbb{R}}|f(x)|<\infty$ and that (2.2) holds for all $x \in \mathbb{R}$. Put $x=\pi$ in (2.2) to get

$$
|f(\pi)+1| \leq \frac{2\left(1+M_{f}\right)}{|d|} \varepsilon .
$$

Furthermore, set $x=y=\pi / 2$ in (2.1) to obtain

$$
\left|f(\pi)-f\left(\frac{\pi}{2}\right)^{2}-d\right| \leq \varepsilon
$$

By combining (3.2) and (3.3), we get

$$
\left|f\left(\frac{\pi}{2}\right)^{2}+d+1\right| \leq \frac{2\left(1+M_{f}\right)+|d|}{|d|} \varepsilon
$$

If we set

$$
c=\sqrt{-d-1}, \quad L=\frac{2\left(1+M_{f}\right)+|d|}{|d|},
$$

then it follows from (3.4) that $\left|f(\pi / 2)^{2}-c^{2}\right| \leq L \mathcal{E}$. Therefore, we can easily check that

$$
\begin{gathered}
\left|f\left(\frac{\pi}{2}\right)-c\right| \leq \sqrt{L \varepsilon} \quad\left(\text { for } c>\sqrt{L \varepsilon}, f\left(\frac{\pi}{2}\right) \geq 0\right), \\
\left|f\left(\frac{\pi}{2}\right)+c\right| \leq \sqrt{L \varepsilon} \quad\left(\text { for } c>\sqrt{L \varepsilon}, f\left(\frac{\pi}{2}\right)<0\right) \\
\left|f\left(\frac{\pi}{2}\right)\right| \leq \sqrt{L \varepsilon+c^{2}} \quad(\text { for } c \leq \sqrt{L \varepsilon}) .
\end{gathered}
$$


Since $\sqrt{L \varepsilon+c^{2}} \leq \sqrt{2 L \varepsilon}$ when $c \leq \sqrt{L \varepsilon}$, we have

$$
\left|f\left(\frac{\pi}{2}\right) \pm c\right| \leq \sqrt{2 L \varepsilon}+\sqrt{L \varepsilon}=(1+\sqrt{2}) \sqrt{L \varepsilon} \quad(\text { for } c \leq \sqrt{L \varepsilon}) .
$$

Hence, it follows that

$$
\left|f\left(\frac{\pi}{2}\right)-c\right| \leq(1+\sqrt{2}) \sqrt{L \varepsilon} \quad\left(\text { for } f\left(\frac{\pi}{2}\right) \geq 0\right)
$$

and that

$$
\left|f\left(\frac{\pi}{2}\right)+c\right| \leq(1+\sqrt{2}) \sqrt{L \varepsilon} \quad\left(\text { for } f\left(\frac{\pi}{2}\right)<0\right) .
$$

Due to (2.2), we now get

$$
\begin{aligned}
|f(x)-c \sin x-\cos x| & \leq\left|f(x)-f\left(\frac{\pi}{2}\right) \sin x-\cos x\right|+\left|\left(f\left(\frac{\pi}{2}\right)-c\right) \sin x\right| \\
& \leq \frac{2\left(1+M_{f}\right)}{|d|} \varepsilon+\left|f\left(\frac{\pi}{2}\right)-c\right|
\end{aligned}
$$

for all $x \in \mathbb{R}$ and

$$
\begin{aligned}
|f(x)+c \sin x-\cos x| & \leq\left|f(x)-f\left(\frac{\pi}{2}\right) \sin x-\cos x\right|+\left|\left(f\left(\frac{\pi}{2}\right)+c\right) \sin x\right| \\
& \leq \frac{2\left(1+M_{f}\right)}{|d|} \varepsilon+\left|f\left(\frac{\pi}{2}\right)+c\right|
\end{aligned}
$$

for all $x \in \mathbb{R}$. Therefore, if $f(\pi / 2) \geq 0$, then (3.10) and (3.8) imply

$$
|f(x)-c \sin x-\cos x| \leq \frac{2\left(1+M_{f}\right)}{|d|} \varepsilon+(1+\sqrt{2}) \sqrt{L \varepsilon}
$$

for all $x \in \mathbb{R}$. Similarly, if $f(\pi / 2)<0$, it then follows from (3.11) and (3.9) that

$$
|f(x)+c \sin x-\cos x| \leq \frac{2\left(1+M_{f}\right)}{|d|} \varepsilon+(1+\sqrt{2}) \sqrt{L \varepsilon}
$$

for all $x \in \mathbb{R}$.

By (2.1) and our hypothesis $0<\varepsilon<|d|$, we have

$$
|f(x)||f(y)| \leq|f(x+y)|+2|d|
$$

for all $x, y \in \mathbb{R}$, which implies that $M_{f}^{2} \leq 2|d|+M_{f}$, and hence

$$
M_{f} \leq \frac{1+\sqrt{1+8|d|}}{2} \text {. }
$$


Subsequently, it follows from (3.15) that

$$
L \leq \frac{3+\sqrt{1+8|d|}+|d|}{|d|},
$$

and hence

$$
\begin{aligned}
& \frac{2\left(1+M_{f}\right)}{|d|} \varepsilon+(1+\sqrt{2}) \sqrt{L \varepsilon} \\
& \quad \leq \frac{3+\sqrt{1+8|d|}}{|d|} \varepsilon+(1+\sqrt{2}) \sqrt{\frac{3+\sqrt{1+8|d|}+|d|}{|d|}} \sqrt{\varepsilon} .
\end{aligned}
$$

Note that if $d<-1$, then the Butler-Rassias functional equation (1.1) has exactly two solutions $\pm c \sin x+\cos x$ (see Theorem 1.2.) Thus, it follows from (3.12), (3.13), and (3.17) that

$$
\left|f(x)-f_{0}(x)\right| \leq \frac{3+\sqrt{1+8|d|}}{|d|} \varepsilon+(1+\sqrt{2}) \sqrt{\frac{3+\sqrt{1+8|d|}+|d|}{|d|}} \sqrt{\varepsilon}
$$

for any $x \in \mathbb{R}$ and for some solution function $f_{0}$ of the Butler-Rassias functional equation. Putting

$$
K=\max \left\{\frac{3+\sqrt{1+8|d|}}{|d|},(1+\sqrt{2}) \sqrt{\frac{3+\sqrt{1+8|d|}+|d|}{|d|}}\right\}
$$

in the last inequality, we conclude that our assertion is true.

Remark 3.2. If we set $d=0$ in the Butler-Rassias functional equation (1.1), then the equation is called the exponential functional equation. Baker, Lawrence, and Zorzitto [2] have investigated the stability problem for the exponential equation (see also [1]).

\section{Acknowledgments}

The author would like to express his cordial thanks to the referee for his/her very valuable suggestions. This work was supported by the 2004 Hong-Ik University Academic Research Support Fund.

\section{References}

[1] J. A. Baker, The stability of the cosine equation, Proc. Amer. Math. Soc. 80 (1980), no. 3, 411416.

[2] J. A. Baker, J. Lawrence, and F. Zorzitto, The stability of the equation $f(x+y)=f(x) f(y)$, Proc. Amer. Math. Soc. 74 (1979), no. 2, 242-246.

[3] S. Butler, Problem no. 11030, Amer. Math. Monthly 110 (2003), 637.

[4] G. L. Forti, Hyers-Ulam stability of functional equations in several variables, Aequationes Math. 50 (1995), no. 1-2, 143-190.

[5] D. H. Hyers, On the stability of the linear functional equation, Proc. Natl. Acad. Sci. USA 27 (1941), 222-224. 
[6] S.-M. Jung, On the Hyers-Ulam stability of the functional equations that have the quadratic property, J. Math. Anal. Appl. 222 (1998), no. 1, 126-137.

[7] On the stability of the Lobačevskil equation, Funct. Differ. Equ. 6 (1999), no. 1-2, $111-$ 123.

[8] M. T. Rassias, Solution of a functional equation problem of Steven Butler, Octogon Math. Mag. 12 (2004), 152-153.

[9] S. M. Ulam, Some questions in analysis, Problems in Modern Mathematics, John Wiley \& Sons, New York, 1964, 63-80, Chapter VI.

Soon-Mo Jung: Mathematics Section, College of Science and Technology, Hong-Ik University, 339-701 Chochiwon, Korea

E-mail address: smjung@wow.hongik.ac.kr 